\title{
Tracking visible and occluded targets: Changes in event related potentials during motion extrapolation
}

\author{
Alexis D.J. Makin, Ellen Poliakoff*, Wael El-Deredy \\ School of Psychological Sciences, University of Manchester, Oxford Road, Manchester, M13 9PL, UK
}

\section{A R T I C L E I N F O}

\section{Article history:}

Received 25 July 2008

Received in revised form 6 January 2009

Accepted 7 January 2009

Available online 16 January 2009

\section{Keywords:}

EEG

ERPs

Movement

Attention

Occlusion

Velocity

\begin{abstract}
A B S T R A C T
Previous research suggests that there is overlap between the systems used for tracking visible and occluded moving targets. Both visually derived and stored velocity information provide input to the tracking system when the target is visible, but after occlusion the system receives its input solely from stored velocity representations. We used EEG to investigate the transition to purely memory guided tracking after the initial period of occlusion. Participants fixated while they covertly tracked targets moving horizontally rightward at velocities of 12 or $20^{\circ} / \mathrm{s}$. The targets were either continuously visible or disappeared for a short interval mid trajectory. Similar positive event related components were recorded over the right occipitoparietal region in both tasks, providing further evidence that the same neural systems are involved in tracking both visible and occluded targets. Furthermore, when the target was visible, the development of this positivity was linked to the spatial location of the target, peaking earlier in the $20 \% \mathrm{~s}$ condition. However, in the occluded condition the positive deflection began around $200 \mathrm{~ms}$ following the onset of occlusion and was not modulated by target velocity or location. This indicates a neural response after occlusion is cortically registered, consistent with the hypothesis that there is increased reliance on memory guided tracking at this point.
\end{abstract}

(c) 2009 Elsevier Ltd. All rights reserved.

\section{Introduction}

It is known that primates can attentively track single, visible moving targets (Krauzlis \& Stone, 1999). However, visuomotor processing requires $100-200 \mathrm{~ms}$ and during this time a target may move significantly (Schlag \& Schlag-Rey, 2002). Predictive pursuit systems are therefore necessary to overcome such delays (Barnes \& Asselman, 1991; Nijhawan, 1994). Different predictive mechanisms, however, may be recruited depending on the nature of the task. For example, one predictive mechanism may be recruited when participants attempt to catch a ball, but another recruited when they verbally report a judgement about its position (Tresilian, 1995). Nevertheless, one consequence of any predictive system is that very brief interruptions in input are covered. In support of this, when a moving target disappears, smooth pursuit eye movements continue at the same velocity during the initial period of occlusion (Bennett \& Barnes, 2003). Similarly, participants' judgements about the location of moving targets are not impaired over occlusion intervals with a duration of less the 200 ms (Benguigui, Broderick, \& Ripoll, 2004). These results can both be explained in the same way: all tracking requires predictive pursuit to overcome delays, so no additional cognitive systems are required during brief occlusion periods.

\footnotetext{
* Corresponding author. Tel.: +44 161275 7333; fax: +44 1612752588 .

E-mail address: Ellen.Poliakoff@manchester.ac.uk (E. Poliakoff).
}

Other studies have investigated the tracking of occluded targets over longer intervals. For example, in motion extrapolation tasks participants typically see a moving target which disappears or becomes occluded, and they then make a judgement about the time it would have reached a particular point further along its trajectory (Rosenbaum, 1975). One possibility is that the attentive mechanisms used to track visible targets are also used to track occluded targets, but during occlusion these systems receive input from stored velocity information. This would allow participants to track the occluded targets fairly accurately, despite the loss of visual feedback (Wexler \& Klam, 2001). This form of memory guided tracking may be employed during occlusion if target reappearance is expected or volitional effort applied (Pola \& Wyatt, 1997). Some evidence for this model comes from recordings of eye movements during motion extrapolation. For example, Bennett and Barnes (2006) found that smooth pursuit eye movement velocity declined after the first 100-200 ms of occlusion and reduced velocity smooth pursuit and saccades were evident for the rest of the occlusion period. These eye movements kept eye position roughly aligned with the position of the occluded target, indicating that oculomotor systems have access to a representation of the occluded targets velocity. It could be that visual feedback and predictive pursuit systems guide tracking of visible targets and during the initial period of occlusion; then stored velocity representations are employed for tracking the occluded target when these inputs are no longer available (although see Barnes \& Collins, 2008 and Section 4). 
Neuroimaging studies have also explored visible and occluded target tracking. For example an fMRI study by Olson, Gatenby, Leung, Skudlarski, and Gore (2004) found that the right medial temporal/medial superior temporal complex (MT/MST, also known as V5/V5a in humans) and bilateral intraparietal sucli (IPS) were both activated by the presentation of either visible or occluded target movement. Lencer et al. (2004) also found bilateral V5 and anterior ventral IPS activation during both visible and occluded tracking, along with V3a, the right posterior IPS and the frontal eye fields (FEFs). In fact, with the exception of V3a and V5, activation was actually greater in the occluded condition. Moreover, there were additional activations of the right supplementary eye fields (SEFs) and the dorsolateral prefrontal cortex (DLPFC) during occlusion. These findings suggest that the same neural systems are involved in tracking visible and occluded targets, but that this pursuit network is enhanced by top-down inputs during occlusion. Nevertheless, fMRI studies lack the temporal resolution to ascertain when this transition occurs.

In the current study, we used scalp recorded event related potentials (ERPs) to investigate the hypothesis that the attentive tracking system is guided by visual velocity signals and top-down velocity information while the target is visible, but by stored velocity representations alone during occlusion. According to this hypothesis, the same attentive tracking systems are involved in tracking visible and occluded motion, so we expected to see comparable topography and morphology of ERPs in the visible and occluded conditions. However, we also predicted that the latency of ERPs recorded on the occluded trials would reflect the transition to purely memory guided tracking after the initial period of occlusion. This should occur at approximately $200 \mathrm{~ms}$ after occlusion onset, when the disappearance of the target has been cortically registered.

Electroencephalography (EEG) data was recorded during two separate tasks in a single session. In both tasks participants fixated while tracking single targets moving horizontally from left to right at 12 or $20^{\circ} / \mathrm{s}$. During the visible tracking task, the target remained visible throughout the trial. During the occluded tracking task, the target became occluded for at least $500 \mathrm{~ms}$. In both tasks, the procedure encouraged attentive tracking of the targets. In the visible task, participants detected oddballs; brief velocity changes that were presented on a minority of trials. In the occluded task, participants judged whether targets reappeared from occlusion at the correct time or too late. In order to perform the occluded tracking task successfully, it was necessary to track the position of the occluded target at the correct velocity, and then assess whether reappearance occurred near to its estimated position. While there is a debate about the forms of information involved in processing occluded motion (Hecht \& Savelsburgh, 2004; Schiff \& Oldak, 1990), the task used here requires visuospatial tracking across the occlusion interval (DeLucia \& Liddell, 1998).

In all four conditions (visible, occluded, $12 \% \mathrm{~s}, 20^{\circ} / \mathrm{s}$ ) the targets covered the same distance as they travelled from left to right. This allowed us to explore the development of ERPs as a function of time and of the spatial location of the targets. If ERPs are linked to time, their amplitudes would be equivalent in the 12 and $20 \% \mathrm{~s}$ conditions at a particular time point, even though target location is more advanced in faster trials. Conversely, if ERPs are linked to the location of the target, their amplitudes would be equivalent in the 12 and $20 \%$ s conditions when the target is at a particular location, even though less time has elapsed in the faster trials.

\section{Method}

\subsection{Participants}

Data from 17 participants aged 19-31 (mean age 23, 8 male, 3 left handed) was analysed. Data from three other participants was excluded due to excessive eye movements (see Section 2.6). Each participant performed the visible and occluded tracking tasks in separate blocks in one session lasting around $1 \mathrm{~h}$. The order of presentation was counterbalanced, however, due to the exclusion of participants, 10 performed the visible tracking task first. The study had local ethics committee approval and testing was performed in accordance with the ethical standards laid down in the 1991 Declaration of Helsinki.

\subsection{Apparatus}

Participants were seated $75 \mathrm{~cm}$ from a $40 \mathrm{~cm}$ (approximately $29.86^{\circ}$ of visual angle)CRT monitor in a darkened room. Visual stimuli were presented using a ViSaGe Visual Stimulus Generator (Cambridge Research Systems). Participants rested their right hand on a button box and responded by pressing down with their index finger in the visible tracking task, or with their index and middle fingers in the occluded tracking task. Head immobilisation was achieved by use of a chin rest.

In both tasks the moving target was a grey disk (RGB $1,1,1$ ) of $6 \mathrm{~mm}$ in diameter $\left(0.45^{\circ}\right)$, presented at eye level. The target was static at $2.4 \mathrm{~cm}\left(3.21^{\circ}\right)$ from the left of the screen for $600 \mathrm{~ms}$ before moving horizontally to the right. The static presentation was included to alert participants that the trial was beginning, and so potentials evoked by target appearance did not overlap with ERPs related to target motion. A fixation cross was located at the centre of the of the targets path throughout the trial. The inter-trial interval was randomized between 3400 and $4400 \mathrm{~ms}$.

Continuous EEG was recorded using Synamps (Neuroscan Inc) from $61 \mathrm{AgCl}$ extended $10-20$ scalp electrodes relative to a $\mathrm{CZ}$ reference, and subsequently average referenced offline. Vertical and horizontal electro-oculograms were recorded with separate electrodes placed above and below the left eye and on the outer canthi of both eyes respectively. Impedance was kept below $5 \mathrm{k} \Omega$ throughout and EEG was sampled at $500 \mathrm{HZ}$. Bandpass filters were set at $0.01-100 \mathrm{~Hz}$.

\subsection{Visible tracking procedure}

The visible tracking task comprised 20 practice trials and 112 experimental trials. There were an equal number of trials where the targets traversed the screen at velocities of 12 and $20^{\circ} \mathrm{s}$. Sixteen of the trials were velocity change oddballs, which suddenly doubled in speed for $100 \mathrm{~ms}$ at an unpredictable point during their trajectory (Fig. 1, middle panels). Participants were instructed to fixate on the central cross rather than follow the moving target with their eyes and to press the button if they saw a sudden velocity change.

The target trajectory was always $35.25 \mathrm{~cm}$ (26.24 of visual angle), centred on the fixation point, lasting $2186 \mathrm{~ms}$ for $12^{\circ} / \mathrm{s}$ trials and $1314 \mathrm{~ms}$ for $20^{\circ} / \mathrm{s}$ trials. In other words, target displacement was controlled; 12 and $20 \% \mathrm{~s}$ targets travelled the same distance. The point where the velocity change occurred in the oddball trials was randomised between $\pm 11.7 \mathrm{~cm}\left(17.5^{\circ}\right)$ around the central fixation point. This corresponds to a time window of $1458 \mathrm{~ms}$, beginning $364 \mathrm{~ms}$ after the onset of movement on $12 \% \mathrm{~s}$ trials and a time window of $875 \mathrm{~ms}$ beginning after $219 \mathrm{~ms}$ on $20^{\circ} / \mathrm{s}$ trials. Trials were presented in a pseudorandom order. However, for every trial, the parameters of the immediately preceding trial were controlled (cf. Makin, Poliakoff, Chen, \& Stewart, 2008), so 12 and $20^{\circ}$ /s trials were preceded equally often by trials of each velocity, and by an equal number of oddballs. This necessitated the inclusion of two additional trials at the start of each block which were not analysed. The task was split into two blocks of 53 and 59 trials. The order in which blocks were presented was varied between participants. Ten and seven participants were presented with each order.

\subsection{Occluded tracking procedure}

In the occluded tracking task, participants completed 32 practice trials and 160 experimental trials. As in the visible tracking task, target velocity was 12 or $20^{\circ} / \mathrm{s}$ After a period of visible movement, the target disappeared for an interval in the middle part of its trajectory before reappearing either on-time or $300 \mathrm{~ms}$ too late (Fig. 1, lower panels). The participants were instructed to fixate rather than follow the target with their eyes, and to maintain fixation even when the target became invisible. They were told that the task was to judge whether reappearance was ontime or too late, assuming that the target had travelled at a constant velocity during occlusion. They reported their judgements by pressing a button with their index finger to report correct reappearance and another button with their middle finger to report late reappearance. We did not include an early reappearance condition in order to maximise the occlusion duration and to avoid general expectation of early reappearance. This minimised possible overlap between expectation and tracking during the first period of occlusion.

The target trajectory was always $35.25 \mathrm{~cm}\left(26.24^{\circ}\right)$, centred on the fixation point. The first visible period was $8 \mathrm{~cm}\left(10.72^{\circ}\right)$ in length and thus lasted $500 \mathrm{~ms}$ for $12^{\circ} / \mathrm{s}$ targets and $300 \mathrm{~ms}$ for $20^{\circ} / \mathrm{s}$ targets. The targets were then occluded for one of four equally spaced distances: $13.7 \mathrm{~cm}\left(10.21^{\circ}\right), 15.2 \mathrm{~cm}\left(11.37^{\circ}\right), 16.8 \mathrm{~cm}\left(12.54^{\circ}\right)$ or $18.4 \mathrm{~cm}\left(13.71^{\circ}\right)$. This results in four levels of occlusion duration for on-time $12 \%$ targets $(850,950,1050$ and $1150 \mathrm{~ms})$ and the on-time $20^{\circ} / \mathrm{s}$ targets $(510,570,630$ and $690 \mathrm{~ms}$ ). The presentation of different levels of occlusion duration reduced the likelihood of participants learning standard occlusion durations over the course of the experiment (cf. Jones \& Wearden, 2004). After occlusion, the target reappeared and continued moving rightward until it reached the end of its trajectory. With the late trials, targets reappeared $300 \mathrm{~ms}$ later than in the on-time trials. It should be noted 


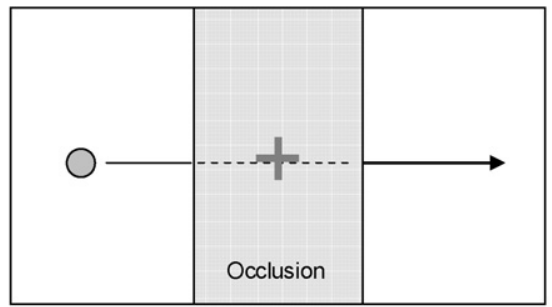

\section{Visible tracking}

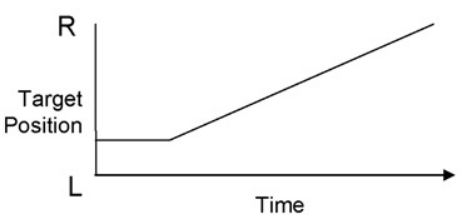

\section{Occluded tracking}

Correct Reappearance

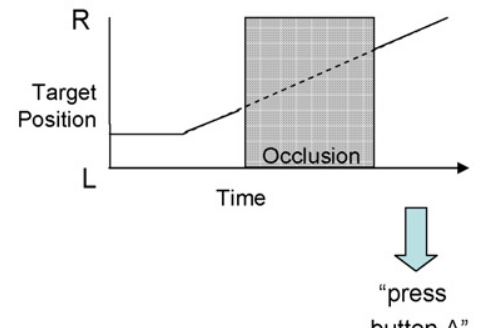

Oddball Trial

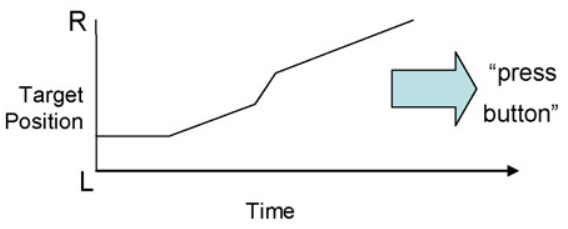

Late Reappearance

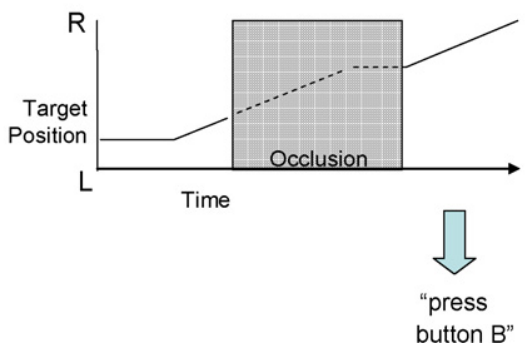

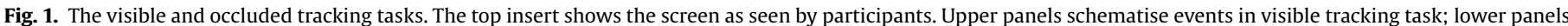



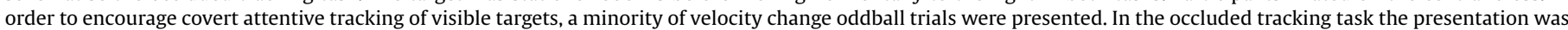

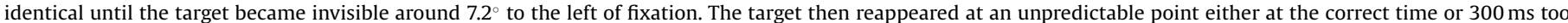

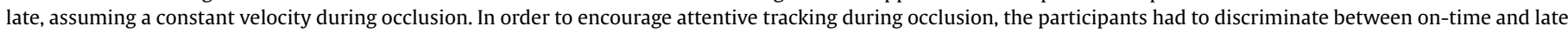
trials.

that the stimuli in the visible and occluded tasks were identical until the onset of occlusion in the occluded task. This allowed direct comparison between ERPs relating to visible and occluded motion. In the on-time occluded trials, the targets after reappearance were identical to those in the visible trials.

There were 80 trials of each velocity $\left(12,20^{\circ} / \mathrm{s}\right)$ and 80 at each level of reappearance (on-time, late). As with the visible tracking task, the immediately preceding trial was controlled, so every type of trial was preceded by an identical distribution of trial types (Makin et al., 2008). This necessitated the inclusion of five additional trials, at the start of each block, which were not analysed. The occluded task was split into five blocks of 32-34 trials, with a break between each block. Five different block orders were arranged according to a Latin square and three or four participants carried out each order.

\subsection{Analysis of behavioural data}

Signal detection analysis was used to assess performance in the visible task. This procedure provides a measure of sensitivity to a stimulus which is not always present, as well as the bias towards reporting the presence of the stimulus (Green \& Swets, 1966). In the occluded task the proportion of trials judged to have reappeared at the correct time analysed as a function of actual reappearance with a repeated measures ANOVA. Data were not available from 2 of the 17 participants due to a problem recording the button press responses. The EEG data from these participants, however, was included.

\subsection{Analysis of EEG data}

Artefacts in the EEG data resulting from eye blinks and mains interference were removed using independent components analysis (ICA) (Jung et al., 2000) and segmented into epochs $400 \mathrm{~ms}$ before target onset to $3092 \mathrm{~ms}$ after target appearance. Epochs were baseline corrected relative to a pre-target onset period of $200 \mathrm{~ms}$.

Trials with horizontal eye movement within the first $1700 \mathrm{~ms}$ of target onset were excluded from analysis. Horizontal eye movement was identified by the ampli- tude exceeding $70 \mu \mathrm{V}$ at electrodes AF7 or AF8, or by a correlation of $>0.75$ between AF7/AF8 and the horizontal electro-oculogram. The loss of trials was $9.14 \%(M=10.23$ trials, S.D. $=5.16)$ in the visible task and $17.57 \%(M=28.11$ trials, S.D. $=14.89)$ in the occluded tracking task.

This difference was significant $(t(16)=3.504, p=0.003)$, suggesting that it was more difficult to fixate in the occluded task. However, it should be noted that the trials included in the EEG analysis all met identical criteria. In addition, 1.73\% ( $M=1.941$ trials, S.D. $=1.60)$ and $2.059 \%(M=3.29$ trials, S.D. $=2.14)$ of trials were lost in the visible and occluded tracking tasks respectively due to a failure of the apparatus to detect the trigger signal. Three participants were excluded from the analysis because fewer than $50 \%$ of their trials remained after excluding trials with eye movements. Data from each condition was averaged for each of the remaining 17 participants and used for statistical analysis.

To explore the patterns of ERP activity during visible and occluded tasks, sequences of topographic maps of scalp activity were produced (Praamstra, Boutsen, \& Humphreys, 2005), using group mean voltage at each electrode, averaged over a 40 ms time window. The visible tracking task was explored as follows: first topographic maps were selected from time points evenly spaced across the $20 \% \mathrm{~s}$ target trajectory. These were compared to topographic maps from the $12 \% \mathrm{~s}$ conditions in which the target had been visible for exactly the same amount of time. This produced the time-aligned sequence. Next we produced the location-aligned sequence. For this, the same points were used for the $20 \% \mathrm{~s}$ condition but were matched to points in the $12 \%$ s condition when the target was at the same location. Time-aligned and location-aligned sequences were also produced for the occluded tracking tasks, but these were limited to the occlusion period only. Here time refers to the amount of time elapsed since the target became occluded. Finally additional, sequences were produced for the visible tracking task, but realigned according to the point of occlusion onset in the occluded tracking task (subsets of these time-aligned and location-aligned sequences are shown in Fig. 2).

This visual analysis identified clusters of electrodes for further exploration. Here we explore the right sided occipitoparietal electrodes (P2, P4, P6, P8, PO4, P08, O2) and their left sided homologues. Frontal activity was also analysed to assess the 
Visible
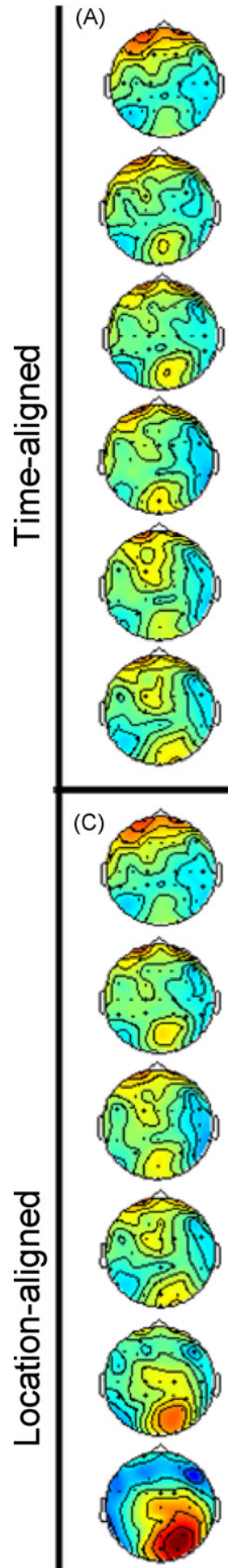

$12 \%$
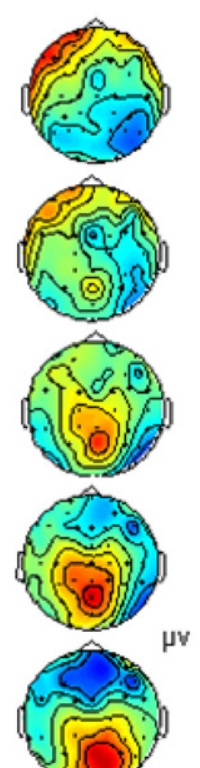

$\mu v$

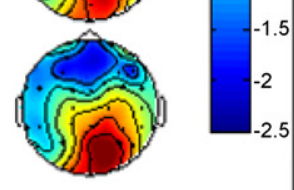

(B)


$300 \mathrm{~ms}$
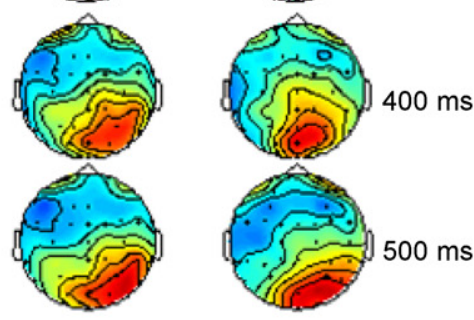

(D)
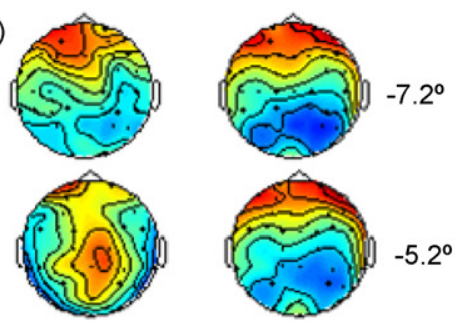

$-5.2^{\circ}$
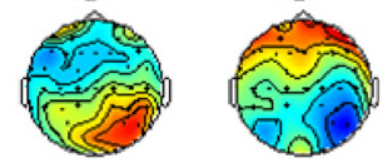

$-3.2^{\circ}$
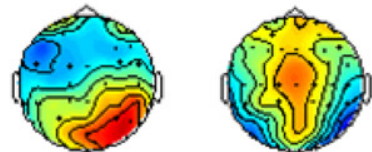

$-1.2^{\circ}$
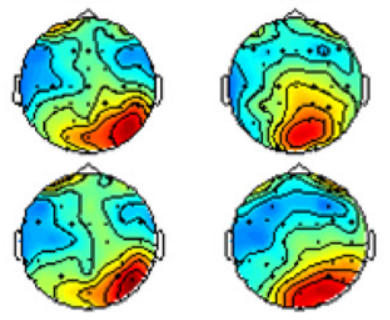

$0.8^{\circ}$

$12 \%$

$20 \% / \mathrm{s}$

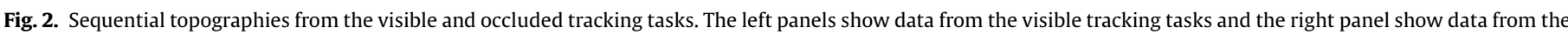

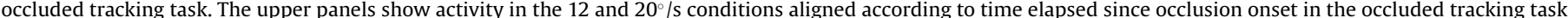

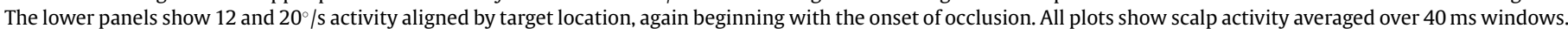

role of unwanted eye movements. Electrodes FP2, AF4, AF8 and F8 and their left sided homologues were used for this. Parietal-central activity was also analysed to explore the role of motor preparation relating to the button press response. This analysis involved electrodes $\mathrm{C} 2, \mathrm{C} 4, \mathrm{C} 6, \mathrm{CP} 2, \mathrm{CP} 4, \mathrm{CP} 6$ and their left sided homologues. Repeated measures ANOVAs were used to compare mean amplitude in the left and right electrode clusters at different points. In all ANOVAs, the amplitude at a particular time point was the average amplitude over a $40 \mathrm{~ms}$ window around the stated point. Significant interactions were explored with paired samples $t$ tests. The Greenhouse-Geisser correction factor was used when sphericity was violated. 
Table 1

ANOVA analysis of left and right occipitoparietal electrodes in the visible tracking task.

\begin{tabular}{lccccl}
\hline & Left & & & \multicolumn{2}{l}{ Right } \\
\cline { 2 - 3 } \cline { 6 - 6 } & $F$ & $p$ & & $F$ & $p$ \\
\hline Velocity & 1.997 & 0.177 & & $<1$ & NS \\
Time & 2.481 & 0.069 & & 16.969 & $<0.001$ \\
Interaction & 10.568 & $<0.001$ & & 6.738 & 0.001 \\
Velocity & $<1$ & $\mathrm{NS}$ & & 2.185 & 0.159 \\
Location & 6.000 & 0.002 & & 17.081 & $<0.001$ \\
Interaction & 2.496 & 0.048 & & 1.796 & 0.152 \\
\hline
\end{tabular}

\section{Results}

To explore the EEG data from the visible and occluded tracking tasks, sequences of topographic maps were produced. A subset of these can be seen in Fig. 2: the time-aligned topographies show six time points for the 12 and $20^{\circ} / \mathrm{s}$ conditions (upper panels) and the location-aligned topographies show the voltage at each electrode while the target was at six locations (lower panels). Both sequences begin at the time of occlusion onset in the occluded tracking task. The data shown here is referenced to the $20 \%$ s condition: that is, the time-aligned and location-aligned sequences are the same in the $20 \%$ s condition. There are three key features of this data. First, there was an increase in positivity over right occipitoparietal regions in both the visible and occluded tasks. This topographic specificity was confirmed statistically. ${ }^{1}$ This activity is our main concern henceforth, and thus the right occipitoparietal ERPs are shown in Fig. 3A-D (see Fig. 3F for the exact position of this cluster).

Second, in the visible tracking task an asymmetry between the 12 and $20^{\circ} / \mathrm{s}$ conditions emerged with time in this region (Figs. $2 \mathrm{~A}$ and $3 \mathrm{~A}$ ). It can be seen that the right occipitoparietal positive deflection peaks earlier in the $20^{\circ} / \mathrm{s}$ condition than the $12 \%$ s when participants track visible targets. The location-aligned topographies show a different pattern, with right occipitoparietal activity in 12 and $20^{\circ}$ /s conditions developing in parallel (Figs. 2C and 3C). This suggests that the development of right occipitoparietal positivity was related to the location of the target in the visible tracking task.

Third, in the occluded tracking task, the right occipitoparietal positivity developed with time in both the 12 and the $20 \%$ s conditions (Figs. 2B and 3B). Conversely, this component became evident

\footnotetext{
1 Two statistical approaches were used to explore the topographic distribution of ERP activity. First we established that ERP activity in the right occipitoparietal cluster was different to that in other scalp regions. Mean ERP activity in the right occipitoparietal electrode cluster was statistically compared to the activity from five other electrode clusters. We analysed amplitude at time points from 0 to $2200 \mathrm{~ms}$ after motion onset in $100 \mathrm{~ms}$ increments. The right occipitoparietal cluster used in this analysis is defined in Section 2.6. The other electrode clusters were (1) the left occipitoparietal cluster as defined in Section 2.6, (2) the right frontal region [FP2 AF4 AF8 F2 F4 F6 and F8], (3) the left sided homologues of these electrodes, (4) the right central region [FC2 FC4 FC6 C2 C4 C6, CPZ CP4 and CP6], (5) the left sided homologues of these electrodes. This data was obtained for each condition $\left(12 \% \mathrm{~s}\right.$ visible, $20^{\circ} / \mathrm{s}$ visible, $12 \% \mathrm{~s}$ occluded and $20 \% \mathrm{~s}$ occluded). Significant Time $\times$ Region interactions were found in all analyses $(F(3.192,51.077)>3.211, p<0.028)$, confirming that the development of activity in the right occipitoparietal region was distinct from that in the other electrode clusters. Secondly, we wanted to confirm that the right occipitoparietal peaks all had a similar topography. For each condition we obtained average voltage in every electrode over the $600 \mathrm{~ms}$ when the right occipitoparietal ERP occurred. Values in each condition and electrode were then normalized by (1) taking the amplitude at each electrode from and subtracting minimum amplitude at any electrode, (2) subtracting the maximum amplitude at any electrode from the minimum amplitude at any electrode, and (3) dividing the result of 1 by the result of 2 . This procedure eliminates amplitude differences between conditions which would otherwise bias topographic analysis (McCarthy and Wood, 1985). The normalized values were the entered into a 2-factor repeated measures ANOVA [4 (condition) by 61 (electrode)]. There was no condition by electrode interaction $(F(8.504,136.072)=1.560, p=0.138)$, suggesting a similar topographic distribution across all conditions. Note, however that the validity of this technique has been questioned (Luck, 2005).
}

when the target was in a more leftward location in the $12^{\circ} / \mathrm{s}$ condition than the $20^{\circ} / \mathrm{s}$ condition (Figs. 2D and 3D). This suggests that right occipitoparietal activity was related to the time elapsed since occlusion onset. To explore these ERPs statistically the right occipitoparietal data from visible and occluded tasks are first analysed separately. This is followed by comparison of data from the occluded and visible tasks over an equivalent period.

\subsection{Visible tracking task}

\subsubsection{Behavioural results}

The 15 participants, for whom data were available, were sensitive to the velocity change oddballs (average $d^{\prime}=2.07$, range $1.21-2.8$ ). A one sample $t$ test found the average $d^{\prime}$ value was significantly greater than zero (chance level) $(t(14)=15.521, p<0.001)$. All participants responding cautiously with a bias towards reporting that no velocity change oddball occurred $(C=0.76$, range $0.2-1.28)$, whether or not an oddball had been presented. That is, even when the distribution of different trial types is taken into account, participants still showed a bias towards reporting 'no velocity change oddball'.

\subsubsection{EEG results}

Repeated measures ANOVAs were used to explore average voltage in the 12 and $20^{\circ} / \mathrm{s}$ conditions as a function of time elapsed since motion onset [Velocity $\left(12^{\circ} / \mathrm{s}, 20^{\circ} / \mathrm{s}\right) \times$ Time $(120,300,480$, $660,840,1020$ and $1200 \mathrm{~ms})]$, and as a function of target location [Velocity $\left(12^{\circ} / \mathrm{s}, 20^{\circ} / \mathrm{s}\right) \times$ Location $(-10.8,-7.2,-3.6,0,3.6,7.2$ and $10.8^{\circ}$ from fixation)]. Results are shown numerically in Table 1 and graphically in Fig. 4. Note that these data sets are exactly the same for the $20^{\circ} / \mathrm{s}$ condition, and that these data points are spaced evenly across the target's trajectory. As with all analyses here, the spacing of these points is necessarily arbitrary: i.e. we could have increased or decreased the number of levels in the time and location factors. However, the number of levels used reflects a reasonable balance between capturing patterns in the ERP data and reducing the risk of spurious findings unrelated to substantial differences in morphology.

This analysis revealed a Velocity $\times$ Time interaction in both hemispheres. On the right, voltage was higher in the $20 \% \mathrm{~s}$ condition at $840 \mathrm{~ms}$ from movement onset $(p<0.001)$ but at $1200 \mathrm{~ms}$ from movement onset it was higher in the $12^{\circ} / \mathrm{s}$ condition $(p=0.024$, Fig. 4B). On the left there was also a significant interaction but no clear cross over effect. Voltage was higher in the $12 \%$ s condition at $840 \mathrm{~ms}(p=0.002)$ and also at $1200 \mathrm{~ms}(p=0.001$, Fig. 4A). Analysis by Location revealed a borderline significant Velocity $\times$ Location interaction on the left ( $p=0.048$, all post hoc tests $>0.05$, Fig. $4 \mathrm{C}$ ) and no interaction on the right ( $p=0.152$, Fig. 4D). The lack of right sided Velocity $\times$ Location interaction contrasts with a highly significant Velocity $\times$ Time interaction in the same region. The pattern of results confirms the impression given by the sequential topographies and ERPs above, and suggests that the development of right occipitoparietal activity was linked to target location during the visible tracking condition.

\subsection{Occluded tracking task}

\subsubsection{Behavioural results}

To assess whether participants were tracking the occluded targets accurately, the proportion of trials judged to be have reappeared at the correct time was analysed as a function of velocity and actual reappearance error with a repeated measures ANOVA [Velocity $\left(12 \%, 20^{\circ} / \mathrm{s}\right) \times$ Reappearance Error (correct, late)]. There was a main effect of velocity $(F(1,14)=10.036, p=0.007)$, and participants were more likely to report correct reappearance on trials which really did reappear at the correct time $(F(1,14)=43.869, p<0.001)$. 

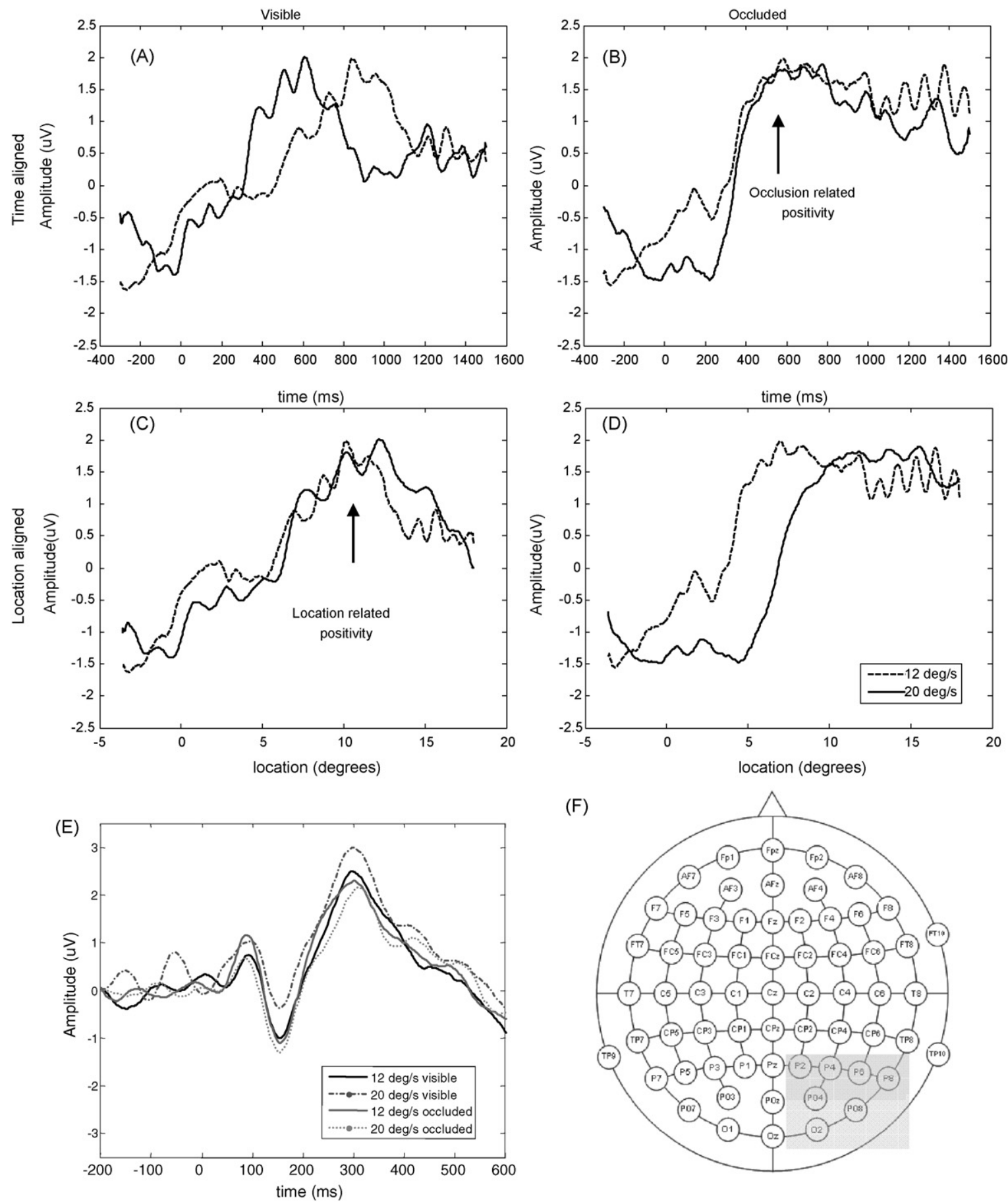

(F)

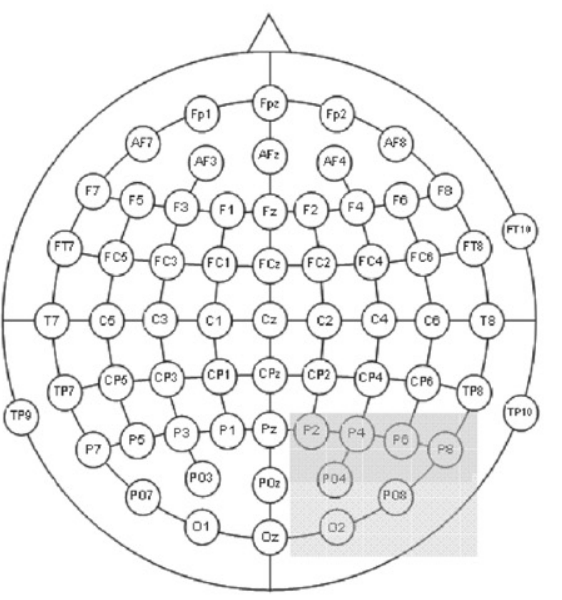

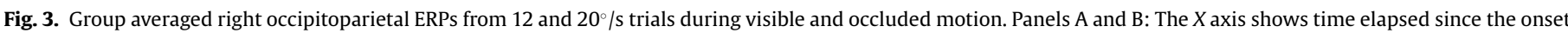

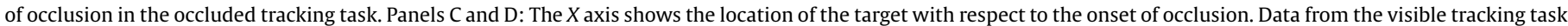



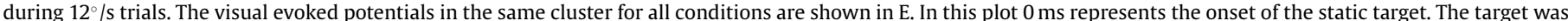

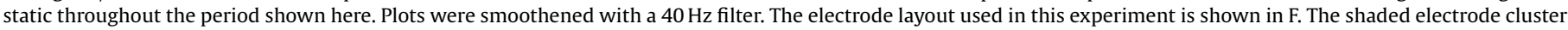
was used to produce these ERP plots.

There was no significant interaction between velocity and reappearance error $(F(1,14)=0.602, p=0.451)$. This was because subjects were similar at discriminating reappearance error in the $20 \% \mathrm{~s}$ condition $\left(t(15)=5.739, p<0.001 ; 20^{\circ} / \mathrm{s}\right.$ on-time $M=0.84$, S.D. $=0.17$; $20^{\circ} / \mathrm{s}$ late $M=0.59$, S.D. $\left.=0.18\right)$ and the $12^{\circ} / \mathrm{s}$ condition $(t(15)=5.640$, $p<0.001 ; 12^{\circ} / \mathrm{s}$ on-time $M=0.71$, S.D. $=0.14 ; 12^{\circ} / \mathrm{s}$ late $M=0.42$, S.D. $=0.18)$. In addition, response times were found to be significantly later on the $12^{\circ} / \mathrm{s}$ trials $(M=3149 \mathrm{~ms}$, S.D. $=342)$ than on the $20^{\circ} / \mathrm{s}$ trials $(M=2476 \mathrm{~ms}$, S.D. $=310 ; t(14)=38.44, p<0.001)$.
This is unsurprising given that reappearance occurred later in $12^{\circ} / \mathrm{s}$ trials.

\subsubsection{EEG results}

To explore the ERPs in the occluded tracking task, data from the occlusion interval rather than the whole trial was analysed. Again left and right occipitoparietal data were treated separately. Two-factor repeated measures ANOVAs were used to compare voltage in the 12 and $20^{\circ} / \mathrm{s}$ conditions at six different post-occlusion 

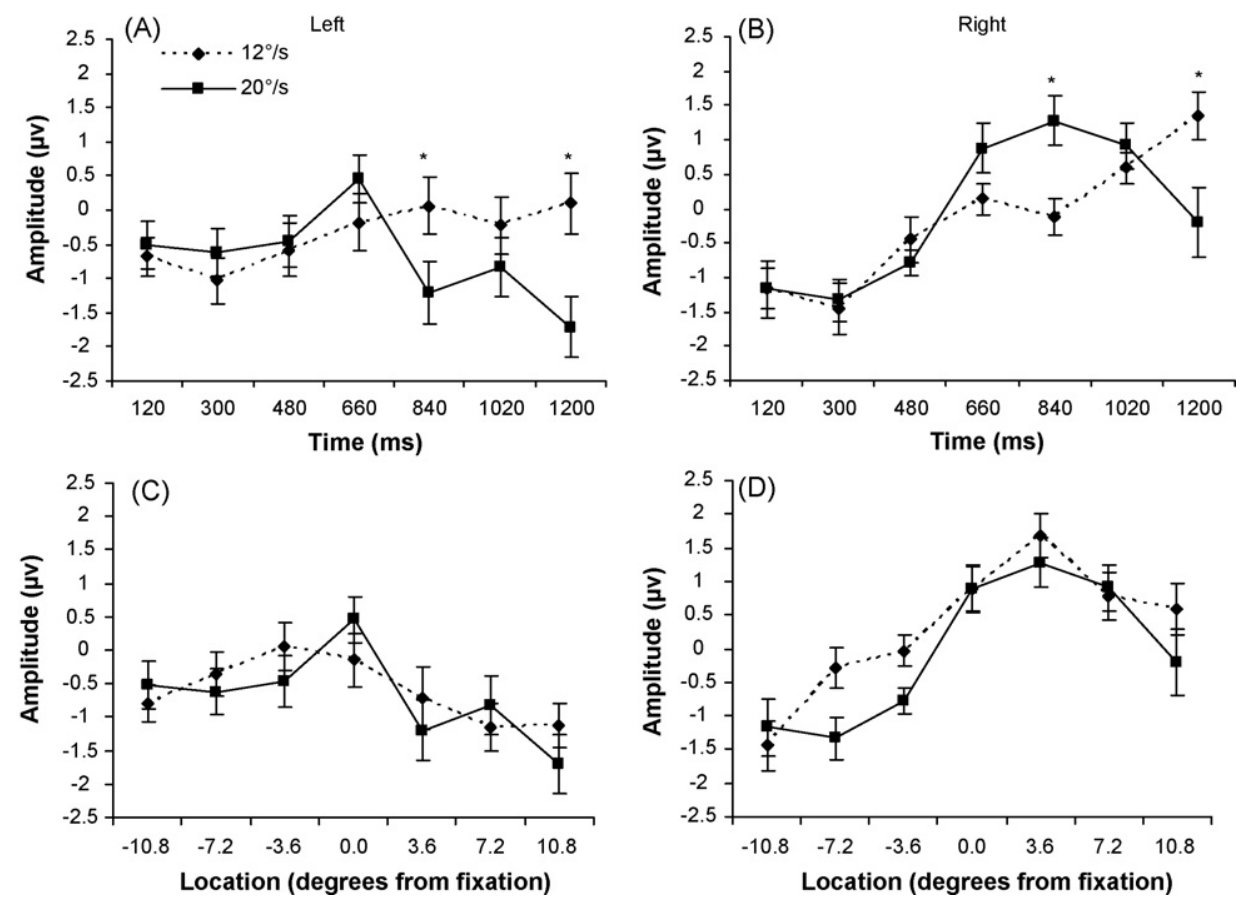

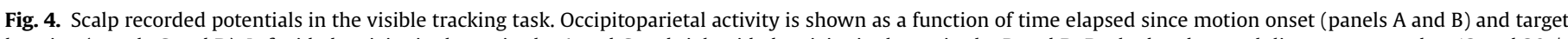

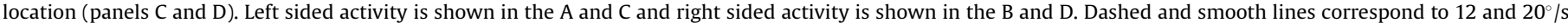
conditions respectively. ${ }^{*}$ indicates a significant voltage difference following a significant interaction. Error bars $= \pm 1$ S.E.M.

time points [Velocity $\left(12^{\circ} / \mathrm{s}, 20^{\circ} / \mathrm{s}\right) \times$ Time $(0,100,200,300,400$, $500 \mathrm{~ms})]$ and when the occluded target was at six different postocclusion locations [Velocity $\left(12^{\circ} / \mathrm{s}, 20^{\circ} / \mathrm{s}\right) \times$ Location $(-7.2,-5.2$, $-3.2,-1.2,0.8$, and $2.8^{\circ}$ from fixation)]. Data is shown in Fig. 5A-D, and in Table 2. Again, the same data was used for the $20^{\circ} / \mathrm{s}$ condition in the time and location analysis.

In the left occipitoparietal region, there were no interactions (Fig. 5A and $\mathrm{B}$ ). In the right occipitoparietal region, both Veloc- ity $\times$ Time and Velocity $\times$ Location interactions were significant. Post hoc $t$ tests revealed a significant difference between the first four time points $(p<0.018)$ but not at $400 \mathrm{~ms}$ post occlusion ( $p=0.173$ ) or at $500 \mathrm{~ms}$ post occlusion $(p=0.828$, Fig. $5 \mathrm{C})$ and between the 12 and $20^{\circ}$ /s conditions when the target was at every occluded location apart from the rightmost ( $p<0.017$, Fig. 5D).

In summary, the pattern of results in occluded task confirmed the impression given by the sequential topographies and ERPs.

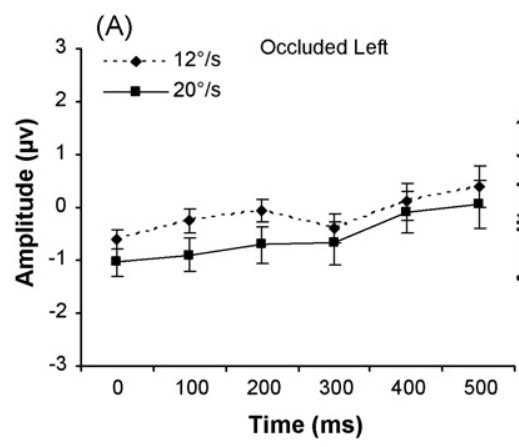

(C)
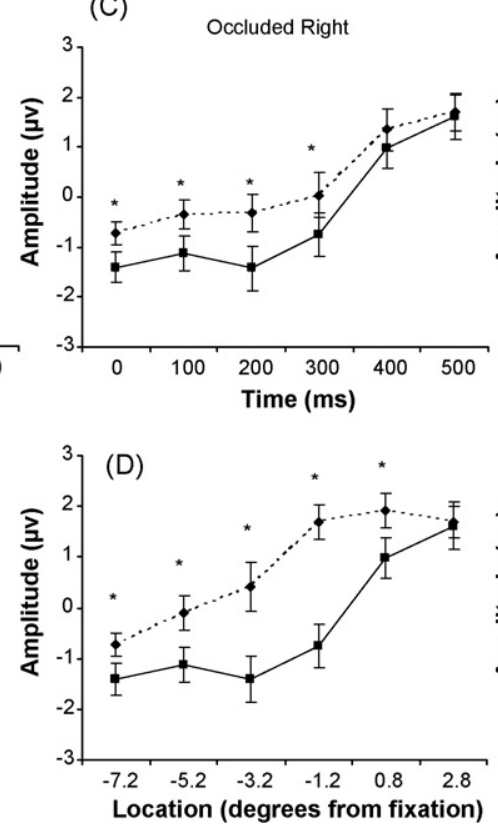

(E)
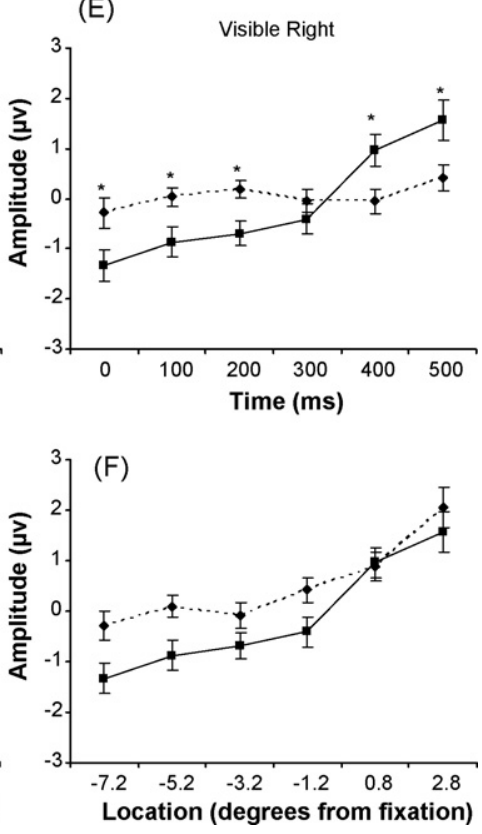

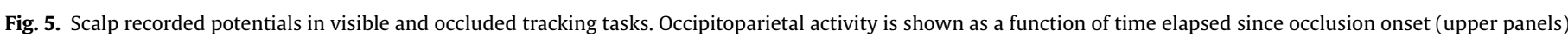

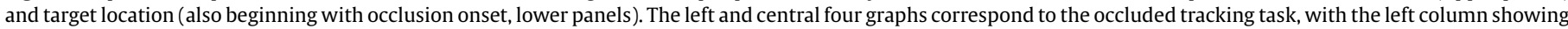

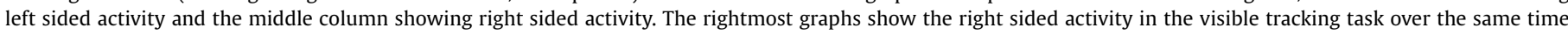

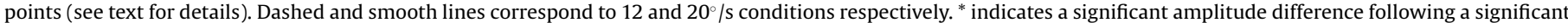
interaction. Error bars $= \pm 1$ S.E.M. 
Table 2

ANOVA analysis of left and right occipitoparietal activity in the occluded tracking task, and analysis of right occipitoparietal activity in the visible tracking task over an equivalent period.

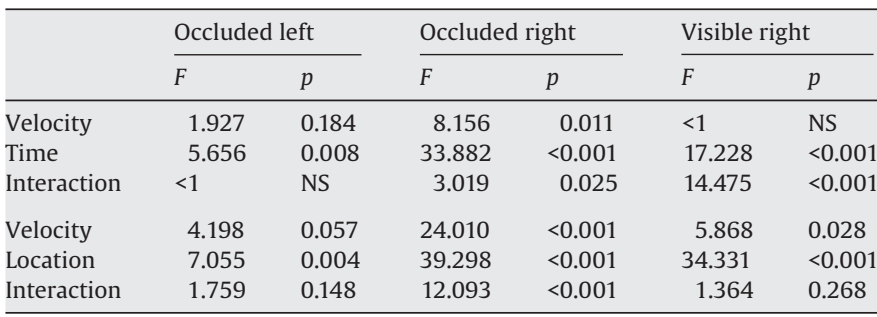

The right occipitoparietal activity becomes increasingly similar between 200 and 400 after occlusion $\mathrm{ms}$ in the 12 and $20^{\circ} / \mathrm{s}$ conditions, and is related to the time elapsed since occlusion onset rather than velocity or the location of the occluded target after this initial period. Although analysis by Location produced an interaction, the pattern of results is quite different. Here voltage in the 12 and $20^{\circ} / \mathrm{s}$ conditions become increasingly different until both had been occluded for $400 \mathrm{~ms}$. There was little systematic activity in the left electrodes in either visible or occluded tasks.

\subsection{Comparison of visible and occluded tasks}

The above analyses of visible and occluded tasks were not conducted on equivalent epochs, which precluded direct comparison. Next, right occipitoparietal activity was compared between the tasks over equivalent intervals. The electrode cluster which defined the right occipitoparietal region is the same as that used in all previous analysis. The post occlusion data used in the above analysis of occluded tracking is compared with identical time points in the visible task. The sequential topographies in Fig. 2 correspond to this analysis. Data is shown in Fig. 5C-F, and in Table 2.

Three way interactions between Velocity, Time and Task, and between Velocity Location and Task were found in both cases ( $F$ $(2.538,41.321)=4.255, p=0.014 ; F(2.78,44.486)=4.933, p=0.006)$. In this narrower analysis of the visible task there was again a Velocity $\times$ Time interaction. There were significant voltage differences between 12 and $20^{\circ} / \mathrm{s}$ conditions at all time points except $300 \mathrm{~ms}$. Voltage was higher in the $12^{\circ} / \mathrm{s}$ condition at 0,100 and $200 \mathrm{~ms}$, but higher in the $20^{\circ} / \mathrm{s}$ condition at 400 and $500 \mathrm{~ms}$ ( $p<0.021$, Fig. 5E). However, there was no Velocity $\times$ Location interaction (Fig. $5 \mathrm{~F}$ ). This pattern replicates the original analysis of the visible tracking task and contrasts with the analysis of the occluded tracking task. This confirms that the interactions between target velocity and time and between target velocity and location were modulated by whether the participants were tracking visible or occluded targets.

The direct comparison between visible and occluded tracking ERPs can be summarised as follows. Firstly, even with this narrower analysis, the right occipitoparietal activity in the visible task was found to be best predicted by target location. The positive deflection recorded by occipital and parietal electrodes peaked after the target passed the centre of the screen. In the $20^{\circ} / \mathrm{s}$ trials, the target reached this position screen earlier that it did in the $12 \%$ s trials. Because of this, there was an interaction between Velocity and Time. This positive waveform can be referred to as the Location-related positivity. In the occluded tracking task a different pattern of right occipitoparietal activity was evident. After $200 \mathrm{~ms}$ of occlusion, similar positive deflections were recorded in the 12 and $20 \%$ s conditions, despite the occluded target being in different locations. The latency of this component was directly related to the onset of occlusion. It can thus be referred to as the Occlusion-related positivity (see labels in Fig. 3).
To further explore these location and occlusion-related ERPs, we measured the latency of these components in each participant. Latency was defined as the time point when amplitude reached $50 \%$ of its peak (Luck, 2005). We analysed latency in the visible and occluded 12 and $20^{\circ} / \mathrm{s}$ trials. There was a significant task by velocity interaction $(F(1,16)=27.528, p<0.001)$. This was due to earlier latency in the $20^{\circ} / \mathrm{s}$ condition $(M=410 \mathrm{~ms}$, S.D. $=145)$ than the $12 \%$ s condition $(M=646 \mathrm{~ms}$, S.D. $=201 ; t(16)=5.74, p<0.001)$ in the visible tracking task, but no difference between the $12 \%$ s condition $(M=359 \mathrm{~ms}$, S.D. $=65)$ and the $20^{\circ} / \mathrm{s}$ condition $(M=344 \mathrm{~ms}$, S.D. $=90 ; t(16)=0.831, p=0.41)$ in the occluded tracking task. This confirms the impression given by the group averaged time-aligned ERPs shown in Fig. 3A and B.

In addition to the main analyses, activity prior to motion onset was investigated to establish data quality. Activity in the right occipitoparietal electrode cluster was explored during the period from $200 \mathrm{~ms}$ before target onset to the $600 \mathrm{~ms}$ after target onset but before movement onset. The visual evoked potential (VEP) produced by target onset is shown in Fig. 3E. To confirm the ERPs were similar across conditions prior to movement onset, we carried out a 2-factor repeated measures ANOVA [nine time points $(-200$, $-100,0,100,200,300,400,500,600 \mathrm{~ms}$ from target appearance of the static target $) \times$ four conditions $\left(12^{\circ} / \mathrm{s}\right.$ visible, $20^{\circ} / \mathrm{s}$ visible, $12 \%$ s occluded, and $20 \%$ s occluded). This analysis was repeated twice using time points displaced from the original by -35 and $-65 \mathrm{~ms}$ to ensure that results were not an artefact of the chosen time points. Together these analyses cover every pre motion onset time point. In all three cases there was a main effect of time $(F(2.202,35.232)>6.564, p<0.003)$, no main effect of condition $(F(1.730,27.686)<2.50, p>0.106)$ and no interaction $(F(6.468$, $103.485)<1.802, p>0.101)$. This suggests that the pre motion onset ERPs were similar in all conditions, suggesting the signal to noise ratio in our data was adequate. The small S.E.M. around the averages used in the main analysis also indicates that our EEG signal was of sufficient quality (see Figs. 4 and 5).

Finally, because our experiment was not fully counterbalanced (10 of the 17 participants did the visible task first), we re-ran all analysis with an additional between-subjects factor of presentation order (visible first vs. occluded first). There were no significant interactions between presentation order and any of the effects reported above $(F(1,15)<1.6, p>0.2)$. This suggests presentation order does not explain the difference between visible and occluded ERPs.

\subsection{Potential confounds}

There are three possible alternative explanations for the pattern of ERPs found in the right occipitoparietal region. Firstly, it is possible that the activity here was produced by electrical activity resulting from unwanted eye movements. However, activity in frontal regions which would be most affected by eye movements did not mirror the effects of interest. There was no three way interaction between Velocity, Time and Task $(F(2.469,39.503)=1.391$, $p=0.261$ ) and only a borderline interaction between Velocity, Location and Task $(F(3.671,50.678)=2.533, p=0.064)$. It should also be noted that most eye movements were removed by the exclusion procedures outlined above. The second potential confound comes from the cortical preparation of the manual button-press responses. There are two arguments against this. Firstly, left parietal-central activity, which would relate to preparation of a right hand response (Walter, Cooper, Aldridge, McCallum, \& Winter, 1964) was not evident. Left parietal-central activity was analysed over the same time points as the effects of interest. In the visible task there was no effect of time $(F(3.2,51.6)<1, \mathrm{NS})$ and although the effect was present in the occluded task $(F(2.5,39.4)=7.989, p=0.001)$, the amplitude of this activity was very low (around $\pm 1 \mu \mathrm{V}$ ). Second, there was a con- 
vergence of positive deflections in the 12 and $20 \% \mathrm{~s}$ conditions in the occluded task, while the average response time in 12 and $20 \%$ trials occurred at significantly different times. This again suggests that our effects were not simply due to manual motor preparation. Finally, it could be argued that the development of ERPs reflects the fact that attention must be sustained for different periods of time in the fast and slow trials. However, this account is incompatible with the precise relationship between ERP amplitude and target location, and does not explain the difference between location and occlusion-related ERPs.

\section{Discussion}

Two tasks were employed to explore the tracking of visible and occluded targets. In the visible tracking task, participants fixated while attentively tracking visible targets from left to right in order to detect rare velocity changes. Signal detection analysis revealed that participants were performing above chance. In the occluded tracking task, identical 12 and $20 \% \mathrm{~s}$ targets were presented, but they became occluded for the central part of their trajectory. The targets then reappeared either at the correct time or too late, and participants had to distinguish between these events. Successful performance necessitated the attentive tracking of the occluded target (DeLucia \& Liddell, 1998); the reappearance point was unknown, so a timing strategy could not be used because participants could not calculate the reappearance time in advance. Participants could discriminate between on-time trials and late trials, at both velocities. This is despite the fact that performance in motion extrapolation tasks declines with occlusion duration (e.g. Lyon \& Waag, 1995; Viana \& Giulianini, 2004), and 20\% s trials were occluded for less time than $12 \% \mathrm{~s}$ trials in this task.

The ERPs recorded here suggest that tracking visible and occluded targets involve overlapping systems. In both visible and occluded tracking tasks, there was an increase in positive potentials recorded by right occipitoparietal electrodes. This topographic similarity is in line with previous fMRI studies, which have also found overlapping activations during visible and occluded motion (Lencer et al., 2004; Olson et al., 2004). Our results also suggest that these tracking systems may be lateralized, as the distribution of scalp activity was highly asymmetrical. This is consistent with observations of greater right sided activations in fMRI studies (Lencer et al., 2004; Olson et al., 2004) and with the right dominance of attentional systems suggested by neuropsychological studies (Heilman, Watson, \& Valenstein, 2002). Indeed, it could be argued that this consistency further supports our assumption that attentive tracking systems were responsible for generating the ERPs recorded here.

Nevertheless, it is unclear whether the lateralization of the location and occlusion-related ERPs was due to the rightward direction of target movement, or due to the anatomical locations of the generators. Other ERPs which have been linked to these brain regions, such as the anterior directing attention negativity (ADAN) and the late directing attention positivity (LDAP) are lateralized according to the direction of the attentive shift (e.g. Eimer, Van Velzen, Gherri, \& Press, 2007). It will therefore be important to measure ERPs associated with leftward motion in future studies.

As highlighted in the introduction, there is a distinction between tracking targets over occlusions with durations shorter than visuomotor delay and tracking targets over longer occlusions. For example, motion extrapolation performance begins to decline at around $200 \mathrm{~ms}$ after occlusion onset (Benguigui et al., 2004) and smooth pursuit eye movements are only interrupted by occlusion after 100-200 ms (Bennett \& Barnes, 2006; Pola \& Wyatt, 1997). The latency of the ERPs recorded in the current study is consistent with this idea. In the visible tracking task, the development of the right occipitoparietal positivity was linked to the location of the moving target, and therefore peaked earlier in the $20^{\circ} / \mathrm{s}$ trials than the $12 \%$ s trials. Conversely, when participants tracked occluded targets, right occipitoparietal positivity peaked at the same time in the 12 and $20 \%$ s conditions. This positive deflection began at around $200 \mathrm{~ms}$ post occlusion, and peaked at around $500 \mathrm{~ms}$, irrespective of the target's location or velocity (see Fig. 3). The latency of this Occlusion-related positivity indicates that there is a change in neural activity after the first period of occlusion, and that this change is time-locked to occlusion onset. Moreover, ERP waveforms in the visible and occluded task were similar prior to the onset of the Occlusion-Related Positivity (confirmed statistically, although analysis not included here for brevity), suggesting that similar visually guided tracking systems were involved prior to this point. This is consistent with the hypothesis that the weighting of different velocity inputs to attentive tracking systems changes after the first $200 \mathrm{~ms}$ of occlusion.

However, there is some uncertainty about the relative contribution of different sources of target velocity information in attentive tracking. It has been argued that once eye velocity reaches target velocity, pursuit is maintained by negative feedback from retinal velocity signals and by positive feedback from efference copy of oculomotor drive (Barnes \& Asselman, 1991; Robinson, Gordon, \& Gordon, 1986). The efference copy loop allows the oculomotor system to overcome visuomotor delay and cover short occlusion intervals (Pola \& Wyatt, 1997). More robust, stored velocity representations may only begin to guide tracking of occluded targets only after predictive pursuit systems and visual feedback are no longer available (Bennett \& Barnes, 2003 and see introduction). A second possibility is that target velocity information is sampled from retinal motion signals very early in the presentation, and then this velocity information guides tracking when the target is visible, as well as after occlusion (Barnes \& Collins, 2008). The latter model eschews the idea of an efference copy loop in predictive pursuit. The current ERP data does not discriminate between these alternative models of visible tracking, but does suggest that there is a change in neural processing after the initial period of occlusion. This could be due to the recruitment of stored velocity representations, the removal of visual feedback, or both.

These models of visible and occluded tracking are based on eye movement data, whereas the ERPs recorded here occurred during fixation. Perhaps models of oculomotor tracking should not be generalised to covert pursuit? However, this theoretical position would be inconsistent with the pre-motor theory of attention, which states that systems involved in guiding spatially orientated actions are also involved in guiding shifts of spatial attention, even when the actions are not executed (Rizzolatti, Riggio, Dascola, \& Umilta, 1987). In support of this, neuroimaging studies have found that covert tracking activates many of the same regions as ocular pursuit (Culham et al., 1998; Ohlendorf, Kimmig, Glauche, \& Haller, 2007), and ERPs during the preparation of saccades and covert attentive shifts are similar (Eimer et al., 2007). Therefore it could be argued that many of the same mechanisms are involved in overt and covert tracking. Indeed, the fact that the Occlusion-Related Positivity temporally corresponds to the point where smooth pursuit eye movements are interrupted suggests that overt and covert pursuit are influenced by stimulus parameters in the same way. Future research could compare ERPs recorded in motion extrapolation tasks and in oculomotor tasks that require velocity memory, such as prior to the initiation of anticipatory smooth pursuit eye movements (cf. Barnes \& Asselman, 1991). This could provide evidence for common velocity processing in the two tasks and a potentially informative link between two historically disparate areas of research.

The ERPs reported here are probably due to neural activity in the distributed attentional frontoparietal network (see Mesulam, 2002 for review). As well as being implicated in tracking of visible and occluded targets, various frontoparietal regions have been impli- 
cated directly in velocity memory. For example, lesions covering the MT/MST have been shown to impair delayed velocity discrimination performance in monkeys (Pasternak \& Merigan, 1994) and humans (Greenlee, Lang, Mergner, \& Seeger, 1995). Moreover, FEF neurons show persistent velocity related activity during occlusion intervals (Barborica \& Ferrera, 2003). Interestingly, Lencer et al. (2004) found activation of the DLPFC during their occlusion conditions. This area is widely linked to working memory function, so its activation could reflect increased guidance of tracking systems by stored velocity representations in the absence of visual input. In a follow up study Nagel et al. (2006) also presented visible and occluded targets, and found that DLPFC activity correlated with the level of disruption to smooth pursuit. This implies that the region is recruited whenever tracking requires additional cognitive inputs. These areas may all have contributed to the location and occlusion-related ERPs recorded here.

Given the anatomically distributed nature of the frontoparietal system, it is possible that cross-talk between components could be achieved by synchronous firing of neural populations at a particular frequency (for review see Jensen, Kaiser, \& Lachaux, 2007). In fact, one recent study using magnetoencephalography found that gamma band activity in area MT is proportional to the level of motion coherence in random dot arrays (Siegel, Donner, Oostenveld, Fries, \& Engel, 2007). This suggests that motion representations encoded in MT may communicate with higher visual areas by virtue of their synchronous output. Another study reported phase synchronization in the gamma band between frontal and right parietal cortex during metal object rotation tasks (Bhattacharya, Petsche, Feldmann, \& Rescher, 2001). Note that this right hemisphere bias is topographically consistent with the ERPs found in the current study. Given the theoretical and empirical work in this field, it would be interesting to explore gamma band activity during visible and occluded target tracking.

In conclusion, we have provided further evidence that overlapping attentive tracking systems are involved in tracking visible and occluded targets, as the topographies of ERPs in visible and occluded conditions were similar. The latency of the Occlusion-related positivity also suggests that there is a change in the velocity signals used to guide attentive tracking after the initial period of occlusion, in agreement with previous psychophysical and eye tracking data.

\section{References}

Barborica, A., \& Ferrera, V. P. (2003). Estimating invisible target speed from neuronal activity in monkey frontal eye field. Nature Neuroscience, 6(1), 66-74.

Barnes, G. R., \& Asselman, P. (1991). The mechanism of prediction in human smooth pursuit eye-movements. Journal of Physiology-London, 439, 439-461.

Barnes, G. R., \& Collins, C. J. S. (2008). The influence of briefly presented randomized target motion on the extraretinal component of ocular pursuit. Journal of Neurophysiology, 99(2), 831-842.

Benguigui, N., Broderick, M., \& Ripoll, H. (2004). Age differences in estimating arrivaltime. Neuroscience Letters, 369(3), 197-202.

Bennett, S. J., \& Barnes, G. R. (2003). Human ocular pursuit during the transient disappearance of a visual target. Journal of Neurophysiology, 90(4), 2504-2520.

Bennett, S. J., \& Barnes, G. R. (2006). Combined smooth and saccadic ocular pursuit during the transient occlusion of a moving visual object. Experimental Brain Research, 168(3), 313-321.

Bhattacharya, J., Petsche, H., Feldmann, U., \& Rescher, B. (2001). EEG gamma-band phase synchronization between posterior and frontal cortex during mental rotation in humans. Neuroscience Letters, 311, 29-32.

Culham, J. C., Brandt, S. A., Cavanagh, P., Kanwisher, N. G., Dale, A. M., \& Tootell, R. B. H. (1998). Cortical fMRI activation produced by attentive tracking of moving targets. Journal of Neurophysiology, 80(5), 2657-2670.

DeLucia, P. R., \& Liddell, G. W. (1998). Cognitive motion extrapolation and cognitive clocking in prediction motion tasks. Journal of Experimental Psychology: Human Perception and Performance, 24(3), 901-914.

Eimer, M., Van Velzen, J., Gherri, E., \& Press, C. (2007). ERP correlates of shared control mechanisms involved in saccade preparation and in covert attention. Brain Research, 1135(1), 154-166.
Green, D. M., \& Swets, J. A. (1966). Signal detection theory and psychophysics. New York: Wiley.

Greenlee, M. W., Lang, H. J., Mergner, T., \& Seeger, W. (1995). Visual short-termmemory of stimulus velocity in patients with unilateral posterior brain-damage. Journal of Neuroscience, 15(3), 2287-2300.

Hecht, H., \& Savelsburgh, G. (2004). Theories of time-to-contact judgment. In: Timeto-contact (pp. 1-11). Amsterdam: Elsevier.

Heilman, K. M., Watson, R. T., \& Valenstein, E. (2002). Spatial neglect. In H. O. Karnath, A. D. Milner, \& G. Vallar (Eds.), The cognitive and neural bases of spatial neglect (pp. 3-30). New York: Oxford University Press.

Jensen, O., Kaiser, J., \& Lachaux, J.-P. (2007). Human gamma-frequency oscillations associated with attention and memory. Trends in Neurosciences, 30(7), 317-324.

Jones, L. A., \& Wearden, J. H. (2004). Double standards: Memory loading in temporal reference memory. Quarterly Journal of Experimental Psychology Section B-Comparative and Physiological Psychology, 57(1), 55-77.

Jung, T. P., Makeig, S., Humphries, C., Lee, T. W., McKeown, M. J., Iragui, V., et al. (2000). Removing electroencephalographic artifacts by blind source separation. Psychophysiology, 37(2), 163-178.

Krauzlis, R. J., \& Stone, L. S. (1999). Tracking with the mind's eye. Trends in Neurosciences, 22(12), 544-550.

Lencer, R., Nagel, M., Sprenger, A., Zapf, S., Erdmann, C., Heide, W., et al. (2004). Cortical mechanisms of smooth pursuit eye movements with target blanking. An fMRI study. European Journal of Neuroscience, 19(5), 1430-1436.

Luck, S. (2005). An introduction to the event-related potential technique. Cambridge, MA: MIT press.

Lyon, D. R., \& Waag, W. L. (1995). Time-course of visual extrapolation accuracy. Acta Psychologica, 89(3), 239-260.

Makin, A. D. J., Poliakoff, E., Chen, J., \& Stewart, A. J. (2008). The effect of previously viewed velocities on motion extrapolation. Vision Research, 48, 1884-1893.

McCarthy, G., \& Wood, C. C. (1985). Scalp distributions of event-related potentials-An ambiguity associated with analysis of variance models. Electroencephalography and Clinical Neurophysiology, 62(3), 203-208.

Mesulam, M. M. (2002). Functional anatomy of attention and neglect: From neurons to networks. In H. Karnath, D. Milner, \& G. Vallar (Eds.), The cognitive and neural bases of spatial neglect (pp. 33-45). New York: Oxford University Press.

Nagel, M., Sprenger, A., Zapf, S., Erdmann, C., Kompf, D., Heide, W., et al. (2006). Parametric modulation of cortical activation during smooth pursuit with and without target blanking. An fMRI study. Neuroimage, 29(4), 1319-1325.

Nijhawan, R. (1994). Motion extrapolation in catching. Nature, 370(6487), 256-257.

Ohlendorf, S., Kimmig, H., Glauche, V., \& Haller, S. (2007). Gaze pursuit, 'attention pursuit' and their effects on cortical activations. European Journal of Neuroscience, 26(7), 2096-2108.

Olson, I. R., Gatenby, J. C., Leung, H. C., Skudlarski, P., \& Gore, J. C. (2004). Neuronal representation of occluded objects in the human brain. Neuropsychologia, 42(1), 95-104.

Pasternak, T., \& Merigan, W. H. (1994). Motion perception following lesions of the superior temporal sulcus in the monkey. Cerebral Cortex, 4(3), 247-259.

Pola, J., \& Wyatt, H. J. (1997). Offset dynamics of human smooth pursuit eye movements: Effects of target presence and subject attention. Vision Research, 37(18), 2579-2595.

Praamstra, P., Boutsen, L., \& Humphreys, G. W. (2005). Frontoparietal control of spatial attention and motor intention in human EEG. Journal of Neurophysiology, 94(1), 764-774

Rizzolatti, G., Riggio, L., Dascola, I., \& Umilta, C. (1987). Reorienting attention across the horizontal and vertical meridians-Evidence in favour of a premotor theory of attention. Neuropsychologia, 25(1A), 31-40.

Robinson, D. A., Gordon, J. L., \& Gordon, S. E. (1986). A model of the smooth pursuit eye-movement system. Biological Cybernetics, 55(1), 43-57.

Rosenbaum, D. A. (1975). Perception and extrapolation of velocity and acceleration. Journal of Experimental Psychology-Human Perception and Performance, 1(4), 395-403.

Schiff, W., \& Oldak, R. (1990). Accuracy of judging time to arrival: Effects of modality, trajectory, and gender. Journal of Experimental Psychology: Human Perception and Performance, 16(2), 303-316.

Schlag, J., \& Schlag-Rey, M. (2002). Through the eye, slowly: Delays and localization errors in the visual system. Nature Reviews Neuroscience, 3(3), 191-200.

Siegel, M., Donner, T. H., Oostenveld, R., Fries, P., \& Engel, A. K. (2007). High-frequency activity in human visual cortex is modulated by visual motion strength. Cerebral Cortex, 17(3), 732-741.

Tresilian, J. R. (1995). Perceptual and cognitive-processes in time-to-contact estimation-Analysis of prediction-motion and relative judgment tasks. Perception \& Psychophysics, 57(2), 231-245.

Viana, L., \& Giulianini, F. (2004). Predicting motion: A psychophysical study. In $\mathrm{H}$ Hecht \& G. Savelsburgh (Eds.), Time-to-contact (pp. 53-65). Amsterdam: Elsevier.

Walter, W. G., Cooper, R., Aldridge, V. J., McCallum, W. C., \& Winter, A. L. (1964) Contingent negative variation: An electrical sign of sensorimotor association and expectancy in the human brain. Nature, 230, 380-384.

Wexler, M., \& Klam, F. (2001). Movement prediction and movement production. Journal of Experimental Psychology-Human Perception and Performance, 27(1), 48-64. 